\title{
FATORES DE RISCO MATERNOS PARA TOXOPLASMOSE NUMA MATERNIDADE PÚBLICA DO NORDESTE DO BRASIL
}

\section{${ }^{* 1}$ Maria Renata Lima Verde Teixeira, ${ }^{2}$ Mateus Dantas Torres, ${ }^{3}$ Wanderson Alves Martins, ${ }^{2}$ Andrea Fernandes Barros de Sousa, ${ }^{4}$ Hyandra Gomes de Almeida Sousa, ${ }^{5}$ Giana Gislanne da Silva de Sousa, ${ }^{6}$ Victor Pereira Lima, ${ }^{7}$ Roberta Araújo e Silva, ${ }^{8}$ Márcio Flávio Moura de Araújo, and ${ }^{9}$ Maria Aparecida Alves de Oliveira Serra}

${ }^{1}$ Enfermeira. Centro Universitário da Grande Fortaleza. Fortaleza, Ceará, Brasil

${ }^{2}$ Enfermeiro. Universidade Federal do Maranhão. Imperatriz, Maranhão, Brasil

${ }^{3}$ Enfermeiro. Mestre em Enfermagem. Universidade Federal do Ceará. Docente do curso de Enfermagem. Centro Universitário Ateneu. Fortaleza, Ceará, Brasil

${ }^{4}$ Nutricionista. Unidade de Ensino Superior do Sul do Maranhão. Imperatriz, Maranhão, Brasil

${ }^{5}$ Enfermeira. Mestranda em Enfermagem.Universidade Federal do Maranhão. São Luís, Maranhão, Brasil

${ }^{6}$ Enfermeiro. Mestrando em Saúde e Tecnologia. Universidade Federal do Maranhão. Imperatriz, Maranhão, Brasil

${ }^{7}$ Enfermeira. Doutora em Enfermagem em Promoção da Saúde. Universidade Federal do Ceará. Docente do Curso de

Enfermagem da Universidade Federal do Maranhão. Imperatriz, Maranhão, Brasil.

${ }^{8}$ Enfermeiro. Doutor em Enfermagem. Universidade Federal do Ceará. Docente dos programas de pós-graduação: Mestrado Acadêmico em Enfermagem - Universidade da Integração Internacional da Lusofonia Afro-Brasileira e Mestrado

Profissionalizante em Saúde da Família - Fundação Oswaldo Cruz. Fortaleza. Ceará. Brasil.

${ }^{9}$ Enfermeira. Doutora em Ciências Médico-Cirúrgicas. Universidade Federal do Ceará. Docente do Programa de Mestrado em Saúde e Tecnologia da Universidade Federal do Maranhão. Imperatriz, Maranhão, Brasil

\section{ARTICLE INFO}

Article History:

Received $17^{\text {th }}$ August, 2019

Received in revised form

$26^{\text {th }}$ September, 2019

Accepted $03^{\text {rd }}$ October, 2019

Published online $30^{\text {th }}$ November, 2019

\section{Key Words:}

Toxoplasmosis.

Congenital toxoplasmosis.

Prenatalcare. Pregnancy.

*Corresponding author:

Maria Renata Lima Verde Teixeira

\begin{abstract}
Introduction: Vertical transmission by Toxoplasma gondii usually occurs in women who are infected for the first time during pregnancy. Screening for toxoplasmosis during pregnancy is one of the measures adopted in our country as a form of prevention. Objective: To investigate maternal risk factors associated with gestational toxoplasmosis in a large maternity hospital located in Fortaleza-Ceará. Methodology: This is an observational, quantitative comparative study conducted in a federal public maternity hospital located in Fortaleza, Ceará, Brazil in 2014. Data collection occurred from January to March 2014, through the analysis of medical records. guided by a data collection instrument. The research project was submitted to the Assis Chateaubriand School Maternity Research Ethics Committee (MEAC), and was approved in the register 513.392 / 2014-14. Results: The sample analyzed was established by high risk pregnant women aged 13 to 40 years. The diagnosis of toxoplasmosis was predominant in the age group between 20 and 30 years. As managers diagnosed with toxoplasmosis reside mainly in the interior of Ceará with statistically significant difference compared to pregnant women residing in the capital. Conclusion: It can be concluded that the analysis analyzed as the variables that were associated with toxoplasmosis were: residing within the state, gestational week between 13 to 27 weeks, normal sharing and using positive IgG serology for toxoplasmosis.
\end{abstract}

Copyright (C) 2019, Maria Renata Lima Verde Teixeira et al. This is an open access article distributed under the Creative Commons Attribution License, which permits unrestricted use, distribution, and reproduction in any medium, provided the original work is properly cited.

Citation: Maria Renata Lima Verde Teixeira, Mateus Dantas Torres, Wanderson Alves Martins et al. 2019. "Fatores de risco maternos para toxoplasmose numa maternidade pública do nordeste do brasil”, International Journal of Development Research, 09, (11), 31494-31497.

\section{INTRODUÇÃO}

A toxoplasmose adquirida durante a gravidez é uma das grandes causas de complicações durante gestação a nível mundial, ocasionada pelos hábitos de vidas e condições do ambiente que são considerados fatores relevantes no que se trata da infecção. A transmissão vertical pelo Toxoplasma gondii ocorre geralmente em mulheres que são infectadas pela primeira vez durante gestação, sendo o último trimestre o período com maior risco de transmissão para o feto, pois nesta 
Tabela 1. Distribuições das variáveis sociais das gestantes com e sem toxoplasmose em Fortaleza, CE, 2014

\begin{tabular}{|c|c|c|c|c|c|c|}
\hline & Gestantes com toxoplasmose & Gestantes sem toxoplasmose & Total & $O R$ & $95 \% \mathrm{IC}$ & p-value \\
\hline $\begin{array}{l}\text { VARIÁ VEIS } \\
\text { Idade }\end{array}$ & $\mathrm{N}(\%)$ & $\mathrm{N}(\%)$ & $\mathrm{N}(\%)$ & & & \\
\hline$<20$ a 30 anos & $44(53,6)$ & $38(46,4)$ & $82(100,0)$ & 1,789 & $0,747-4,28$ & 0,189 \\
\hline$>30$ a 40 anos & $11(39,2)$ & $17(60,8)$ & $28(100,0)$ & & & \\
\hline Localização & & & & & & \\
\hline Capital & $32(41,5)$ & $45(58,5)$ & $77(100,0)$ & 1,681 & $0,130-1,738$ & 0,007 \\
\hline Interior & $23(69,7)$ & $10(30,3)$ & $33(100,0)$ & & & \\
\hline EstadoCivil & & & & & & \\
\hline Casada & $29(45,3)$ & $35(54,7)$ & $64(100,0)$ & 0,637 & $0,297-1367$ & 0,246 \\
\hline Solteira & $26(56,5)$ & $20(43,5)$ & $46(100,0)$ & & & \\
\hline Escolaridade & & & & & & \\
\hline$<8$ anos & $10(29,4)$ & $24(70,6)$ & $34(100,0)$ & 0,556 & $0,194-1,589$ & 0,271 \\
\hline$>8$ anos & $12(42,8)$ & $16(18,2)$ & $28(100,0)$ & & & \\
\hline Tabagismo & & & & & & \\
\hline $\operatorname{Sim}$ & $04(57,1)$ & $03(42,9)$ & $07(100,0)$ & 2,299 & $0,481-0,998$ & 0,287 \\
\hline Não & $29(36,7)$ & $50(60,3)$ & $79(100,0)$ & & & \\
\hline Etilismo & & & & & & \\
\hline Sim & $03(75,0)$ & $01(25,0)$ & $04(100,0)$ & 5,100 & $0,507-51,260$ & 0,128 \\
\hline Não & $30(37,1)$ & $51(62,9)$ & $81(100,0)$ & & & \\
\hline
\end{tabular}

fase os distúrbios neurológicos são comumente gerados (KADHEMI et al., 2019). A variação da prevalência a nível mundial atinge os níveis de 15 a $90 \%$, levando em conta os fatores que podem agravar ou não a infecção pela doença. A depender do local em que se realiza o estudo os valores podem alcançar a variação de $80 \%$ (ROCHA et al., 2014). As manifestações da doença depende da idade gestacional, podendo apresentar problemas graves. Alguns estudos apontam que em média $90 \%$ das mulheres são assintomáticas e o corpo responde efetivamente, se recuperando espontaneamente. Devido poder apresentar uma virulência maior, a depender da cepa, do estado imunológico da gestante e ademais fatores se torna imprescindível o diagnóstico e tratamento precoce a fim de reduzir a infecção fetal e possíveis complicações (SADIQUI et al., 2018). A triagem é feita através da identificação dos fatores de riscos e pela elaboração e execução de atividades preventivas, sendo de fundamental importância meios que visem reduzir a exposição às fontes de infecção, além da sorologia que deve ser realizada, uma vez que atitudes de baixo custo e simples podem minimizar a infecção durante a gestação, principalmente em mulheres que nunca tiveram contato. Para obter êxito nessas ações, se faz primordial conhecer os fatores potenciais associados à infecção (SMIT et al., 2019) O rastreamento da toxoplasmose durante a gravidez é uma das medidas adotadas em nosso país, pelo Ministério da Saúde, como forma de prevenção. As gestantes, ao realizarem o pré-natal passam por um acompanhamento, e dentro desse processo são solicitados exames complementares, sendo um deles a sorologia para toxoplasmose no primeiro trimestre e solicitado novamente no terceiro trimestre, caso a IgGseja negativa no primeiro exame (BRASIL, 2012). No Ceará, não existe um programa de vigilância da toxoplasmose gestacional e congênita, as medidas preventivas adotadas segue o sugerido pelo Ministério da Saúde, onde observamos que a não obrigatoriedade da sorologia para toxoplasmose gera em muitos serviços de saúde desconhecimento, despreparo, dificuldades em correlacionar as condutas preventivas e terapêuticas corretas por parte dos profissionais que atendem as gestantes. Dessa forma, o estudo teve como objetivo investigar os fatores de risco maternos associados à toxoplasmose gestacional em uma maternidade escola de grande porte, referência para gestantes de alto risco, localizada em Fortaleza- Ceará. Certamente, o presente estudo poderá contribuir para o conhecimento da toxoplasmose gestacional, por ser uma temática pouco debatida e para a elaboração de políticas públicas e novas estratégias a respeito da assistência em saúde a gestante e ao recém-nascido, voltadas para a prevenção dos fatores de risco maternos à toxoplasmose nesta região.

\section{METODOLOGIA}

Trata-se de um estudo observacional, quantitativo do tipo comparativo, desenvolvido numa maternidade federal pública localizada em Fortaleza, Ceará, Brasil em 2014. Este serviço de saúde é especializado no atendimento gestantes de alto risco na metrópole brasileira citada. $\mathrm{O}$ estudo foi conduzido com uma amostra conveniente de 110 prontuários de gestantes agrupadas em dois grupos. No primeiro grupo (A) foram incluídas 55 prontuários de gestantes com diagnóstico clínico de toxoplasmose. Por sua vez, no segundo grupo (B) foram incluídos 55 prontuários de gestantes que não apresentavam a doença. Foram incluídas no estudo mulheres, gestantes com sorologia IgMe IgG para toxoplasmose de feto único. A coleta de dados ocorreu no período de janeiro a março de 2014, através da análise de prontuários guiada por um instrumento de coleta de dados. Este contemplava variáveis sociodemográficas (idade, localidade, escolaridade, estado civil, tabagismo e etilismo) e clínicas (história obstétrica, tipo de parto, número de consultas pré-natais, sorologia para toxoplasmose, hematócrito e sumário de urina). Como princípio ético e global, o projeto de pesquisa foi submetido ao Comitê de Ética em Pesquisa da Maternidade Escola Assis Chateaubriand (MEAC), tendo sido aprovado sob registro $513.392 / 2014-14$, conforme as recomendações da resolução $n^{\circ}$ 466/12 do Conselho Nacional de Saúde brasileiro. Os dados coletados foram inicialmente digitados em planilha eletrônica do programa Excel versão 2007 e, em seguida, analisados pelo Software StatisticalPackage for the Social Sciences (SPSS Inc., Chicago, Estados Unidos, versão 20.0). No cálculo da OddsRatio (OR) (razões de chance) em relação ao desfecho foram consideradas significativas quando seu valor foi $>1$. Na análise comparativa das proporções das variáveis entre os grupos A e B foi adotado o teste do Qui-Quadrado. Em todos as inferências estatísticas foi adotado um nível de significância de $5 \%(p<0,05)$.

\section{RESULTADOS}

A amostra analisada foi constituída por gestantes de alto risco com idade entre 13-40 anos, média de 25,8 e desvio padrão de 
Tabela 2. Distribuições das variáveis clínicas das gestantes com e sem Toxoplasmose em Fortaleza, CE, 2014

\begin{tabular}{|c|c|c|c|c|c|c|}
\hline & Gestantes com toxoplasmose & Gestantes sem toxoplasmose & Total & $O R$ & $95 \% \mathrm{IC}$ & p-value \\
\hline VARIÁVEIS & $\mathrm{N}(\%)$ & $\mathrm{N}(\%)$ & $\mathrm{N}(\%)$ & & & \\
\hline \multicolumn{7}{|l|}{ Idade Gestacional } \\
\hline$<13$ a 27 semanas & $12(70,6)$ & $05(29,4)$ & $17(100,0)$ & 3,388 & $1,092-10,510$ & 0,028 \\
\hline$>27$ a 41 semanas & $34(41,5)$ & $48(58,5)$ & $82(100,0)$ & & & \\
\hline \multicolumn{7}{|l|}{ Paridade } \\
\hline Primípara & $25(58,1)$ & $18(41,9)$ & $43(100,0)$ & 1,713 & $0,790-3,714$ & 0,171 \\
\hline Multípara & $30(44,8)$ & $37(55,2)$ & $67(100,0)$ & & & \\
\hline \multicolumn{7}{|l|}{ Aborto } \\
\hline Sim & $12(46,1)$ & $14(53,8)$ & $26(100,0)$ & 0,797 & $0,330-1,928$ & 0,615 \\
\hline Não & $43(51,8)$ & $40(48,2)$ & $83(100,0)$ & & & \\
\hline \multicolumn{7}{|l|}{ Tipo de parto } \\
\hline Parto Normal & $17(56,7)$ & $13(43,3)$ & $30(100,0)$ & 2,989 & $1,148-7,783$ & 0,023 \\
\hline Parto Cesáreo & $14(30,4)$ & $32(69,6)$ & $46(100,0)$ & & & \\
\hline \multicolumn{7}{|l|}{ Pré-Natal } \\
\hline Não houve ou $<5$ consultas & $25(49,0)$ & $26(51,0)$ & $51(100,0)$ & 0,929 & $0,439-1967$ & 0,848 \\
\hline$>5$ consultas & $30(50,8)$ & $29(49,2)$ & $59(100,0)$ & & & \\
\hline \multicolumn{7}{|l|}{ Sorologia para IgG } \\
\hline Reagente & $49(70,0)$ & $21(30,0)$ & $70(100,0)$ & 9,722 & $3,480-27,158$ & 0,000 \\
\hline Não Reagente & $06(19,4)$ & $25(80,6)$ & $31(100,0)$ & & & \\
\hline \multicolumn{7}{|l|}{ Anemia } \\
\hline Sim & $14(41,2)$ & $20(58,8)$ & $34(100,0)$ & 1,555 & $0,479-2,785$ & 0,748 \\
\hline Não & $20(37,7)$ & $33(62,3)$ & $53(100,0)$ & & & \\
\hline \multicolumn{7}{|l|}{ Sumário de urina } \\
\hline Normal & $18(46,2)$ & $21(53,8)$ & $39(100,0)$ & 1,582 & $0,629-3,983$ & 0,329 \\
\hline Bacteriúria & $13(35,1)$ & $24(64,9)$ & $37(100,0)$ & & & \\
\hline
\end{tabular}

6,9 anos de idade. Odiagnóstico de toxoplasmose foi predominante na faixa etária entre 20 e 30 anos $(40 \%)$ e entre as mulheres casadas $(58,1 \%)$. As gestantes com diagnóstico de toxoplasmose residiam principalmente no interior do Estado do Ceará com diferença estatisticamente significante em relação às gestantes que residiam na capital $(\mathrm{p}=0,007)$. Parcela substancial das gestantes dos grupos estudados não era tabagista ou elitista contemplando, respectivamente, somente $8,1 \%$ e $4,7 \%$ da amostra, vide em Tabela 1 . De acordo com as condições clínicas das gestantes avaliadas neste artigo, as variáveis que tiveram associação estatisticamente significante com a toxoplasmose foram: idade gestacional entre 13 e 27 semanas $(\mathrm{p}=0,028 ; \mathrm{OR}=3,38 ; 95 \% \mathrm{IC}=1,092-10,510)$, Parto Normal $\quad(p=0,023 ; \quad$ OR $=2,989 ; \quad 95 \% \mathrm{IC}=3,480-27,158) \quad$ e IgGreagente para Toxoplasmose $(\mathrm{p}=0,000 ; \quad \mathrm{OR}=9,72$; $95 \% \mathrm{IC}=3,480-27,158)$. Ao avaliar a história obstétrica nos dois grupos de gestantes, como paridade, aborto,número de consultas de pré-natal, anemia e bacteriúria não foi encontrado diferenças estatísticas entre os grupos. Também não houve diferenças entre os grupos ao associarmos as variáveis: número de consultas pré-natal e resultados laboratoriais de anemia e sumário de urina.

\section{DISCUSSÃO}

A toxoplasmose é uma zoonose causada pela ingestão dos cistos do Toxoplasma gondiipresentes em alimentos como carnes mal passadas ou crua, leite não pasteurizado, frutas, legumes, e água contaminada (SILVA et al., 2019). A infecção pelo $T$. gondiidurante a gravidez apresenta grandes riscos para o feto devido a possiblidade da transferência transplacentária do protozoário, gerando a Toxoplasmose Congênita (SILVA et al., 2019). Dessa forma, a detecção dos grupos de risco para doenças detectáveis no pré-natal possui grande relevância clínica (BRASIL, 2012). De acordo com a análise realizada, as gestantes mais jovens apresentaram maior prevalência do diagnóstico de toxoplasmose do que as mais velhas. Este achado também foi demonstrado em outros estudos em uma cidade do nordeste brasileiro (COSTA JÚNIORet al., 2010) e em outra região do país (BITTENCOURT, 2012). Outro dado relevante encontrado demonstrou que as gestantes que viviam no interior do Estado do Ceará possuíam mais chance para apresentar a doença durante a gestação em detrimento as que viviam na capital $(\mathrm{p}=0,007 ; \quad \mathrm{OR}=1,681 ; 95 \% \mathrm{IC}=0,130$ 01,738), em consonância com outros estudos (BITTENCOURT, 2012; DIAS et al., 2017). Por outro lado, não houve associação estatisticamente significante entre escolaridade e o diagnóstico de toxoplasmose na amostra estudada $(p=0,271)$. Dessa forma, faz-se necessário avaliar quais outros fatores influenciaram neste resultado, como, por exemplo, as condições de moradia, alimentação e qualidade da água que já foram evidenciadas como fator de risco em outros estudos (DIAS et al., 2017;MAIA, 2019; GOMES et al., 2019). Quanto as variáveis clínicas, foi evidenciado a correlação da idade gestacional, o tipo de parto e a sorologia para IgG com a ocorrência da toxoplasmose nas gestantes.

As gestantes com idade gestacional menor que 13 a 27 semanas apresentaram três vezes mais chance de serem diagnosticadas com toxoplasmose do que as gestantes no terceiro trimestre de gravidez $(\mathrm{p}=0,028 ; \quad \mathrm{OR}=3,388$; $95 \% \mathrm{IC}=1,092-10,510)$. Este achado revela que as gestantes da amostra foram diagnosticadas ainda no primeiro ou segundo trimestre da gestação.

A detecção precoce da toxoplasmose por meio da sorologia é indispensável em um pré-natal (BRASIL, 2012).Apesar das dificuldades de realização do exame em âmbito nacional, o mesmo deve ser realizado no primeiro trimestre pois diminui consideravelmente o nível de acometimento fetal (MOURA et al., 2018; DIAS et al., 2017). Quanto ao tipo de parto, as gestantes com toxoplasmose majoritariamente tinham realizado parto normal $(\mathrm{p}=0,023 ; \mathrm{OR}=2,989 ; 95 \% \mathrm{IC}=1,148$ 7,783). As gestantes com toxoplasmose devem ser encaminhadas ao serviço de referência que, em sua maioria, funciona dentro das maternidades (BRASIL, 2012; SOUZA, 2018). A visita as maternidades e o acompanhamento da gestantes pelos profissionais de referência da gestação de alto 
risco pode aumentar a confiabilidade, segurança e acolhimento das gestantes favorecendo que estas realizem o parto normal (SOUZA, 2018; POPOLLI et al., 2018). Dessa forma, supõese que o fato das gestantes com o diagnóstico de toxoplasmose terem sido acompanhadas no serviço de referência pode ter influenciado na decisão das mesmas quanto ao parto normal. $\mathrm{Na}$ análise do exame imunocitoquímico para toxoplasmose, as gestantes com sorologia reagente para IgG apresentaram nove vezes mais chance de possuírem o diagnóstico para toxoplasmose do que o grupo com IgG não reagente $(p=0,000$; $\mathrm{OR}=9,722 ; 95 \% \mathrm{IC}=3,480-27,158)$. Este achado diverge do esperado que determina o grupo que ainda não obteve a primoinfecção como o mais susceptível à doença (MAIAet al., 2019). No entanto, o resultado apontado pelo presente estudo pode estar evidenciando a cronicidade da toxoplasmose nas gestantes, uma vez que apresentar o IgG reagente para toxoplasmose não garante a imunidade total da doença de acordo com resultados encontrados em outros estudos (NASCIMENTO ET AL., 2017;COSTA JÚNIORet al., 2010; BITTENCOURT et al., 2012).

O presente estudo possui limitações tais como possuir uma amostra pequena, conveniente, oriunda de um único serviço, com dados baseados no registro de prontuários das gestantes internadas.Dessa forma, a generalização dos seus resultados em relação à população geral fica prejudicada. No entanto, mesmo com as limitações supracitadas, esta pesquisa foi importante para que os profissionais de saúde conheçam os principais fatores de risco para toxoplasmose que acometem a região do estado do Ceará, visto que a maternidade onde se realizou o presente estudo e referência no atendimento a gestante de alto risco de todo o Estado. A toxoplasmose é um problema de saúde pública, devido à inter-relação existente entre os diversos fatores de risco desencadeantes desse evento. Assim, principalmente quando se tem o risco iminente de toxoplasmose congênita, não se pode trabalhar com a hipótese de que apenas um fator foi o responsável pelo abortamento do feto, partos prematuros, crianças com anomalias graves, mas sim considerá-lo um fator com múltiplas causas que podem ser independentes ou interdependentes. Dessa forma, novos estudos em outras regiões do Brasil, com amostras mais significativas são necessários para ampliar a discussão e trazer novos esclarecimentos sobre este tema.

\section{Conclusão}

Com base nos resultados deste estudo, pode-se concluir que na amostra analisada as variáveis que tiveram associação com a toxoplasmose foram: residir no interior do Estado, semana gestacional entre 13 a 27 semanas, parto normal e possuir sorologia IgG positiva para toxoplasmose.

\section{REFERÊNCIAS}

Bittencourt L.H.F.B. et al., Soroepidemiologia da toxoplasmose em gestantes a partir da implantação do Programa de Vigilância da Toxoplasmose Adquirida e Congênita em municípios da região oeste do Paraná. Rev. BrasGinecolObstet, Londrina (PR), 2012. DOI: http://dx.doi.org/10.1590/S0100-72032012000200004.

Brasil. Ministério da Saúde. Secretaria de Atenção à Saúde. Departamento de Atenção Básica. Atenção ao pré-natal de baixo risco / Ministério da Saúde. Secretaria de Atenção à Saúde. Departamento de Atenção Básica. - Brasília : Editora do Ministério da Saúde, 2012. 318 p.: il. - (Série
A. Normas e Manuais Técnicos) (Cadernos de Atenção Básica, $n^{\circ}$ 32). Disponível em: http://bvsms.saude.gov.br/ bvs/publicacoes/cadernos_atencao_basica_32_prenatal.pd $\mathrm{f}$

COSTA Junior CEO, Monteiro CH. Perfil sorológico da toxoplasmose na grande João Pessoa/ PB. RBAC, 42(2). 149-1154, 2010. DOI: 10.21877/2448-3877.201600388

DE CÁSSIA POPOLLI, Eet al., Vinculação da gestante com a maternidade: a influência no tipo de parto. Enfermagem Brasil, 17 (3), p. 199-207, 2018. Disponível em: https://portalatlanticaeditora.com.br/index.php/enfermage mbrasil/article/view/2404/3739

DIAS, V.A. ORTIZ, M. A. L. Toxoplasmose na gestaçãocausas e consequências. Revista uningáreview, 29(1). 2018. Disponível em: http://revista.uninga.br/index.php/ uningareviews/article/view/1920

GOMES, C. B. et al., Eatinghabits of pregnant Brazilian women: an integrative review of the literature. Ciência \& Saúde Coletiva, v. 24, n. 6, p. 2293-2306, 2019. DOI: http://dx.doi.org/10.1590/1413-81232018246.14702017

Khadem, S. Z.et al., 2019 Prevalence and Risk Factors of Toxoplasma gondii InfectionamongPregnantWomen in HormozganProvince, South of Iran. Iran J Parasitol. 14(1), 167-173. Disponível em: https://www.ncbi.nlm. nih.gov/pmc/articles/PMC6511600/

MAIA, A.O., Aspectos epidemiológicos da toxoplasmose em gestantes atendidas nas unidades básicas de saúde do município de Santa Cruz-RN. 2019. Dissertação de Mestrado. Brasil. Disponível em: https://repositorio. ufrn.br/jspui/handle/123456789/27449

MOURA, D.S. OLIVEIRA, R.C.M MATOS-ROCHA, T.J. Toxoplasmose gestacional: perfil epidemiológico e conhecimentos das gestantes atendidas na unidade básica de saúde de um município alagoano. Arquivos Médicos dos Hospitais e da Faculdade de Ciências Médicas da Santa Casa de São Paulo, 63(2) p. 69-76, 2018. DOI:https://doi.org/10.26432/1809-3019.2018.63.2.69

NASCIMENTO, T.L. PACHECO, C.M. DE SOUSA, F.F. Prevalência de Toxoplasma gondii em gestantes atendidas pelo Sistema Único de Saúde. Ciência \& Saúde,10(2), p. 96-101, 2017. DOI:http://dx.doi.org/10.15448/1983652X.2017.2.23297

Rocha, L. Z. et al., 2014. Sorologia para toxoplasmose em gestantes e récem-nascidos em Santo Antônio da Patrulha, Rio Grande do Sul. ClinBiomed Res. 34(4), 366-370. Disponível em: https://seer.ufrgs.br/hcpa/article/ view/50329

Sadiqui, S. et al., 2018. Distributionof Toxoplasma gondii Ig Mand Ig Gantibodyseropositivity among age groups and gestational periods in pregnantwomen. F1000Res.7(1823). DOI: https://doi.org/10.12688/f1000research.15344.3

Silva, B. C. T. et al. Toxoplasmose congênita: estratégias de controle durante o pré-natal. Cadernos da MedicinaUNIFESO, 2 (1) 2019. Disponível em: www.revista. unifeso.edu.br > index.php > article > download

Smit, G. S. A. et al., 2019. Sero-epidemiological status and risk factors of toxoplasmos is in pregnantwomen in Northern Vietnam. BMC InfectDis. 19(329). Published 2019 Apr 18. Smitet al. BMC InfectiousDiseases. 19(329). DOI:https://doi.org/10.1186/s12879-019-3885-7

SOUZA, L. C. et al. Expectativas de gestantes adolescentes relacionadas à assistência no pré-natal e parto. 2018. Tese de Doutorado. Disponível: https:/www.arca.fiocruz.br/ handle/icict/30937 